\title{
Optimization Methods in Modern Transportation Systems
}

\author{
Ivana HARTMANN TOLIĆ, Goran MARTINOVIĆ, Dominika CRNJAC MILIĆ
}

\begin{abstract}
One of the greatest challenges in the public transportation network is the optimization of the passengers waiting time, where it is necessary to find a compromise between the satisfaction of the passengers and the requirements of the transport companies. This paper presents a detailed review of the available literature dealing with the problem of passenger transport in order to optimize the passenger waiting time at the station and to meet the requirements of companies (maximize profits or minimize cost). After a detailed discussion, the paper clarifies the most important objectives in solving a timetabling problem: the requirements and satisfaction of passengers, passenger waiting time and capacity of vehicles. At the end, the appropriate algorithms for solving the set of optimization models are presented.
\end{abstract}

Keywords: algorithms; analytical models; mathematical models; optimization methods; passenger waiting time; public transportation; timetabling; vehicle scheduling

\section{INTRODUCTION}

In modern transportation systems, the greatest challenge is to define the best timetable by minimizing energy consumption and maximizing economic, technological and social goals. The timetabling and scheduling problem of public transportation is known for years [1-3]. In the presented literature, the goals are to find better algorithms and achieve better results using various methods and known algorithms. The planning of urban passenger transport is a highly complex task and usually observed in two different phases [1]. The first observed phase is minimum passenger waiting time (PWT) at the station and the second is the optimal number of vehicles. In recent years, the train timetabling problem (TTP) is studied and the main problem is determining periodic or non-periodic timetable which satisfies the capacities of the vehicles and limits of operations [4-7]. Some of the greatest challenges in PWT problem algorithms are planning of the optimal number of vehicles and their scheduling, minimizing travel time and minimizing travel distance [8].

Planning of the public transportation network is a complex task that includes four basic sub-problems, which are line planning, timetable development, vehicle scheduling and crew scheduling [9-11]. The subproblems are interconnected and should not be considered separately. In practice, it means achieving the satisfaction of passengers and transport company's satisfaction, i.e. maximizing passenger services and minimizing operational cost $[12,13]$.

The paper presents methods, models and optimization algorithms used in public transportation and published in the scientific literature in recent years regarding PWT at the station and efficiency of the vehicles. Owing to the great amount of theoretical data published in scientific literature, the most important objectives should be chosen as well as the respective algorithm to solve them. Next, the paper discusses the presented theoretical background and clarifies the most appropriate solutions for the latter problem. Some of the presented methods and models are explained in detail and some of them mentioned, because of understanding and getting related conclusions about the problems.

The paper is organized as follows: Section 2 presents an overview of models developed for solving public transportation problems with relevant algorithms. In Section 3, the comparison between methods and algorithms, with classification and summary of related problems presented in the current literature is performed.

\section{AN OVERVIEW OF MODELS DEVELOPED FOR SOLVING THE PROBLEMS OF PUBLIC TRANSPORTATION}

In order to classify the optimization models for minimization of the PWT, in the following subsections, the most relevant methods, the objective functions and algorithms are considered. Subsection 2.1 contains an overview of analytical models for solving public transportation problem. Mathematical models for minimizing the total travel time and for minimizing the PWT at the transfer station are presented in subsections 2.2 and 2.3 as well as relevant algorithms. Mathematical models for minimizing the delay of the vehicles and mathematical models for calculating the optimal time between two consecutive vehicles are presented in subsections 2.4 and 2.5

\subsection{Analytical Models for Solving Public Transportation Problems}

In order to increase the productivity and efficiency of transport services, on one hand, and customer satisfaction on the other hand, four main analytical methods for determining the number of vehicles $f_{j}$ needed in the relevant period $\mathrm{j}$ are presented in $[1,3,10]$. The first two methods are based on the number of vehicles:

$f_{j}=\frac{P_{j}}{\gamma_{j} \cdot c}$

In the first method, $P_{j}$ is the average maximum daily occupancy of the vehicle during the period $j$. In the second method $P_{j}$ is the average maximum hourly occupancy of the vehicle during the period $j$. The product of the entering factor and the vehicle capacity $\left(\gamma_{j} \cdot c\right)$ is the desired vehicle occupancy.

The first two methods are enhanced with the rate of the vehicle load function during the time period $\mathrm{j}$ (number of passengers multiplied by kilometres) $\left(\gamma_{j} \cdot c\right)$ and the 
route length $(L)$ multiplied by desired vehicle occupancy $\left(d_{j}\right)$ from which follow the third and the fourth methods defined as

$$
f_{j}=\max \left[\frac{A_{j}}{d_{j} \cdot L}, \frac{P_{j}}{c}\right]
$$

The third method ensures that the vehicle capacity will never be overdrawn. The fourth method has the additional constraint on total route distance having loads more than the desired occupancy.

The analysis of the average passenger waiting time (APWT) (scheduled and random) at the combined station of feeder bus service and intercity transportation as well as the model for numerical calculating of the APWT at the station with the capacity constraint is presented in [14]. The assumption is that the arrival of the feeder bus service is normally distributed.

APWT for intercity vehicle is

$$
\begin{aligned}
& W=\int_{0}^{H}(N(t)) \cdot\left(1-m_{1}\right) \cdot(H-t) \mathrm{d} t+ \\
& +\sum_{i=1}^{S} \int_{0}^{H}(N(t)) \cdot m_{r} \cdot[(r+1) \cdot H-t] \mathrm{d} t
\end{aligned}
$$

where $N(t)$ is density function of arrival time of passengers at the station according to schedule, $H$ represents headway intercity vehicles and $m_{r}$ is the percentage of missed vehicles for $r^{\text {th }}$ waiting period. For a more precise representation of passengers waiting at the station in intercity transport, it is inevitable to consider the reliability of supply services, passenger behaviour and characteristics of the intercity transportation system.

Most of the methods approach the problem of public transportation from the viewpoint of costs and improvement of the business of transport companies, but other influences such as passenger satisfaction vehicle frequency and distance from the station should be considered as well [15].

The model for finding the optimal number of vehicles or headways with the necessary capacity of the vehicles for the observed period is presented in [16]. They consider passengers who failed to get into the vehicle and then the possibilities are: waiting for the next vehicle or use other means of transportation. From the standpoint of the company and in order to keep passengers, the penalties in the case when the passengers could not enter into the vehicle and tend to leave the station are reduced and on the other hand, the PWT for another vehicle is limited. This analytical model is calculated on the example of the bus route in Auckland, New Zealand and results with a cost reduction of $12.3 \%$ for the morning peak load and $19.5 \%$ for the afternoon.

In the model for scheduling the balanced public transportation, passengers that sit or stand in a vehicle (passenger wants to sit if travel is longer), temporal distribution demand and congestion transportation network are considered [17]. Transit assignment model is useful to assess the number of passengers that use the public transportation. An analytical model that can be used for planning the timetable of transportation services with respect to minimizing the total vehicle delay or maximizing profit is proposed.

\subsection{Mathematical Models for Minimizing the Total Travel Time}

The aim of the public transportation in big cities is to satisfy the economic, environmental and social goals. In order to ensure fast and energy efficient urban rail transport, a nonlinear problem of minimizing the total travel time and minimizing energy consumption is shown in [18]. The model includes the time of arrival/departure of vehicles from the station and time of transferring to another vehicle. By using the latter model they want to reduce the cost (number of trains and power consumption) and improve passenger satisfaction (reduce PWT and the number of transfers, thereby reducing the total travel time). They use sequential quadratic programming (SQP) and evolutionary algorithms to solve the problem of scheduling, and they are compared with the traditional fixed vehicle schedule. SQP method gives better indicators of control and computational complexity in comparison to the genetic algorithm.

\subsection{Mathematical Models for Minimizing the PWT at the Transfer Station}

The model for creating timetable problem using multicriteria approach, with an emphasis on minimizing the PWT at the station is proposed in [19] and [20]. Two criteria: empty seat penalty (empty seat kilometres or empty seats hours) and approximate waiting time at the station are studied. In order to find the appropriate timetable, the problem is started as finding the best path in the graph with weighted edges $c_{i j}=\left(c_{i j}^{1}, c_{i j}^{2}\right) \in \mathbb{N} \times \mathbb{N}$. Using the multiobjective label-correcting algorithm results in a reduction of PWT by $43 \%$ with the acceptable load of the vehicles, according to the timetable.

The problem of the interchange waiting time is defined using timetable synchronization problem (TTSP). The problem can often be solved using the branch and bound (B \& B) method [21]. In order to speed up the time of execution in case of major problems, an optimization based heuristic method (OHM) is developed, which is compared with the B\&B method [22]. Peak and non-peak loads are studied. The cases "just miss" are observed in order to reduce the number of cases when the train just left the station and the passenger was unable to enter. The goal function of their TTSP is to minimize the total travel time of passengers who have interchange. Their OHM is faster and better optimizes the problem because they consider the time spent on the transfer station (walking time between trains). For minimizing interchange waiting time of all passengers on the subway, mixed integer programming optimization model is developed.

Mathematical models in which the objective function is the PWT at the transfer station are developed as mixed integer programming (MIP) models as well [23]. In the first model, the output variable is the departure time of the vehicle at the first station, the second model additionally deals with stopping time of the vehicles at transfer 
stations which is used as a variable set for relaxation of the first model because each line has constant headway.

The equation of the PWT at the transfer station of the passengers who transfer from line $i$ to line $j$ is

$$
\begin{aligned}
& W T_{i j}^{s}=\left(X_{j}+t_{j}^{s}+d t_{j}^{s}+h_{j} Y 2_{i j}^{s}\right)- \\
& -\left(X_{i}+t_{i}^{s}+t t_{i j}^{s}+h_{i} Y 1_{i j}^{s}\right)
\end{aligned}
$$

where $X_{i}$ and $X_{j}$ are departure times of the vehicles from first station in lines $i$ and $j, t t_{i j}^{s}$ is time variable that represents the time that passengers spent on walking from vehicle on line i to vehicle on line $j, h_{i}$ is headway on line $i$ (in minutes), $X_{i}+t_{i}^{s}+t t_{i j}^{s}$ is arrival time of passengers at vehicle in line $j$ from line $i$ at transfer station $s$ and $X_{j}+t_{j}^{s}+d t_{j}^{s}$ is departure time of the first vehicle in line $j$ from the station $s$.

Product $h_{j} Y 2_{i j}^{s}$ is added to the departure time of the vehicle in line $j$ for considering the transferring waiting time of the other vehicle in line $j$. Product $h_{i} Y 1_{i j}^{s}$ is added to the arrival time of the passengers, because of shorter transferring waiting time.

The objective function of the model is the sum of the interchange waiting time of all pairs of lines and for all stations and it is defined as

$$
\min z=\sum_{s} \sum_{i j}\left(\frac{D}{h_{i}} p_{i j}^{s} A W T_{i j}^{s}\right)
$$

where $A W T_{i j}^{s}$ is average transfer waiting time, $\frac{D}{h_{i}}$ is the number of vehicles arriving at transfer station. Complexity of the model is $N K^{2}$, where $N$ is the number of transfer stations, and $K$ is the number of lines in transportation network. The model can be solved using classic MIP solver, such as CPLEX solver $(\mathrm{B} \& \mathrm{~B})$ if there are less than 50 transportation lines.

The objective function of the second model is

$$
\min z=\sum_{s} \sum_{i j}\left(\frac{D}{h_{i}} p_{i j}^{s} A W T_{i j}^{s}\right)+\sum_{s} \sum_{i}\left(\frac{D}{h_{i}} p_{i}^{s} e d t_{i}^{s}\right)
$$

where $e d t_{i}^{s}$ is the time that each vehicle in the line $i$ can spend at the station, which is limited by maximum waiting time. CPLEX solver and genetic algorithm (for the greater real-life network) are used to solve the second model. Genetic algorithm can be used for smaller and larger network as well.

In order to minimize the transfer waiting time, the mathematical model that maximizes the number of parallel vehicles arriving at the station in dependence on the desired number of passengers and period of day is proposed in [9]. This represents a nonlinear and mixedinteger linear programming problem which is an NP problem and the best results are obtained using heuristic algorithms. For the purposes of defining the schedule and reducing operating costs of transport companies, a small number of transfer stations is proposed in [2].

Minimization of the interchange waiting time in the periodic railway timetable scheduling problem (PRTS) is solved using the improved differential evolution (DE) algorithm with dual population [24]. The goal of optimization is to make a schedule of outgoing time for each train at each station so that the interchange waiting time at the transfer station is minimized as opposed to the traditional model which uses a fixed schedule. The APWT is considered as objective function and is generalized for all transfer stations in transportation network. The latter model is compared to a B\&B method and greedy-based heuristic algorithm for solving PRTS problems. [24].

The timetable problem is usually based on the maximization of the number of synchronized vehicles arriving at the transfer station or on the minimization of the total PWT at the station for the vehicle with which the passenger continues his journey [25]. The authors have proposed a stochastic integer programming model to minimize the cost of the total PWT for three types of passengers: transferring passengers, boarding passengers and through passengers. The objective function is to minimize the total expected cost and waiting time i.e., minimize the sum of all waiting time costs in the transportation network for transferring passengers, boarding passengers and through passengers. For solving the latter problem the genetic algorithm with local search is used. The model was applied to a small bus network and cost of waiting time is reduced averagely by $9.5 \%$.

The synchronized timetable optimization model for optimization of PWT is proposed in [26]. Minimizing the maximum of interchange waiting time or reducing the worst interchange time is proposed. Performance indicators are used to check the efficiency of the model. The latter synchronized timetable model can be used to improve the network of public transport and solutions with numerical and genetic algorithm are presented.

Phase-regular scheduling (PRS) method that divides the day into a few periods of equal length is shown in [27]. The stations are all marked with $1,2, \ldots, k$, and the trains have the same speeds between stations. Number of planned vehicles during one period ( $\tau=1$ hour) is marked with $x(\tau)$ while a binary variable that indicates the type of the train (larger of smaller) is

$$
y(\tau)=\left\{\begin{array}{l}
1, \text { largetrain } \\
0, \text { otherwise }
\end{array}\right.
$$

The time between two trains is constant and given by $60 / x(\tau)$ with headway constraint $\left[h_{\min }, h_{\max }\right]$, where $h_{\min }$ and $h_{\max }$ are minimal and maximal intervals between two consecutive trains. Two hypotheses are proposed. In the first model passengers uniformly arrive at the station, so expected APWT is given by $30 / x(\tau)$. The second hypothesis is that the passengers arrived at the station in the particular period have to be transported by another train in the same period according to the timetable. The goal of the model is to minimize the PWT at the station and the cost of occupancy of the vehicle is 
$\min Z=\sum_{\tau \in \mathbb{R}}[F(\tau)+W(\tau)]$

where is $W(\tau)$ total waiting time over a period $\tau$ is

$$
W(\tau)=\sum_{i=1}^{k-1} \sum_{s=1}^{k-i} \frac{30}{x(\tau)} D^{i, i+s}(\tau)
$$

and the occupancy cost is

$$
\begin{aligned}
& F(\tau)=\sum_{j=1}^{x(\tau)} \sum_{i=1}^{k-1} Q_{j}^{i}(\tau) R^{i} \delta \\
& \cdot\left\{Q_{j}^{i}(\tau)-\left[y(\tau) C_{1}+(1-y(\tau)) C_{0}\right]\right\}
\end{aligned}
$$

where $\delta(\tau)$ is sign function that is equal to 1 if $u>0$, and 0 otherwise; $R^{i}$ is the necessary travel time between stations $i$ and $i+1 ; C_{0}$ and $C_{1}$ are capacities of smaller and greater vehicles respectively. The occupancy of the vehicle $\left(Q_{j}^{i}(\tau)\right)$ is the sum of the passengers that remained in the vehicle $j$, at the station $i$ and passenger entered in the vehicle $j$ at the moment $\tau$. The hybrid genetic algorithm is used for solving the problem on the example of intercity railway line in China consisting of five stations [27].

The problem of minimizing the cost of PWT at the station of the Madrid railway is presented in [28, 29] and solved using

$$
\min \delta \sum_{i \in S \backslash\{n\}} \sum_{t \in T \backslash\{p\}} f_{i}^{t}
$$

where $f_{i}^{t}$ is the conservation of the flow of the passengers who wait at the station $i$ at the time $t$

$$
f_{i}^{t}=f_{i}^{t-1}+\sum_{j \in S, j>i} d_{i j}^{t}-u_{i}^{t}
$$

The conservation of the flow in computer networks implies that the sum of all flows equals zero. The variable $d_{i j}^{t}$ is a total number of passengers, waiting or not waiting at the station, who plan to travel from station $i$. In the second formulation of the problem the constraints for speed and waiting at the train station are neglected. The elapsed time between the moment when the $k^{\text {th }}$ train left stations $i$ and $i+1$ is observed. The third formulation of the problem is based on the previous formulation of the problem except that the restrictions are on the departure time from station $\mathrm{i}$ and the minimal and maximal speed and waiting times. Branch and cut algorithm is used for all of the presented models [28]. In comparison to the actual cases the APWT is improved using computational experiments by $30-77 \%$. The total speed and the PWT at the station help to achieve better conditions for objective function and execution time. Adaptive large neighbourhood search (ALNS) algorithm capable of solving large problems and more realistic cases in a short execution time is presented in [29]. In comparison to the previous research solutions regarding to the APWT, it is concluded that the ALNS algorithm satisfies in the case with a large number of cases, while using the B\&B algorithm only solves the problem of minimizing the PWT at the station. Reduction of APWT at the station is $26 \%$ in comparison to CPLEX algorithm.

The same problem is solved using hybrid genetic algorithm relating to a greedy method and local improvement algorithm as shown in [30, 31]. Since genetic algorithms are methods of optimization and imitate random processes in nature, they are used for the optimization of single criteria problems. To enable the cost-benefit between the two criteria, fuzzy logic controller is developed. For a small network and problems with periodic driving time the exact methods are relevant, such as B\&B. Periodic railway scheduling is observed as well. Methods are successful and the PWT at the transfer station is reduced by $7 \%$.

Multicriteria optimization of energy conservation in underground and surface railways and the PWT at the station is shown in [32]. First, they want to maximize the used energy, and minimize the loss of energy between trains at the same station (incoming breaking train creates electricity and the system returns the energy to the network and uses it for the acceleration of the outgoing trains). At the same time they want to minimize the PWT with appropriate restrictions (time between trains, the stopping time of the train at the station, total travel time). Multicriteria model is defined and the properties of Pareto optimality are used. Genetic algorithm is the most commonly used algorithm for solving the integer programming problem in the transportation system. It is concluded that the presented model saves $8.86 \%$ of energy and reduces the PWT of $3.22 \%$ compared with the actual timetable.

\subsection{Mathematical Models for Minimizing the Delay of the Vehicles}

Time spent at the station is the key element in the assessment of service quality and optimization of passenger transportation. The problem occurs if there is a delay in the timetable. The problem can be resolved if a timetable includes extra time for possible delays.

Minimizing the cost function of the PWT, including driving time and sensitivity test, is shown in [33, 34]. In order to minimize the waiting cost function in the case of transferring with the train delay, the best buffer time model is proposed. The delay distribution of incoming train and weighting of the PWT the buffer time is based on. The LP objective function is defined as a cost function of the PWT depending on the buffer time in the form of a linear decision model. It is solved using linear programming. In a small network the PWT cost is reduced by $40 \%$.

A great problem in rail network is the delay of trains due to the traffic jam (caused by accidents, etc.). The PWT is increased as well as the delay of the next train.

The problem of finding a possible timetable to minimize train delays while maximizing total satisfaction is shown in [35]. The problem is defined graphically and computationally. The computational problem is solved using a heuristic algorithm real-system data. 


\subsection{Mathematical Models for Calculating the Optimal Time between Two Consecutive Vehicles}

The optimal departure time of vehicles from station for each line in order to reduce the PWT is fundamental for the optimization of the timetable. The waiting time and walking time during transfer is considered. The model distinguishes direct transfer and the transfer that includes a walk [36]. The objective function of the model is to minimize the cost of the PWT as the bi-level minimization problem. The problem is solved using heuristics, e.g. tabu search algorithm.

An optimization model of the train schedules depending on the requirements of passengers is based on headway equation, the equation of passengers and the dwell time equation which is defined as a function of the amount of passengers who enter and exit the vehicle [37]. The goal of the optimization problem is to minimize the PWT at the station and operating cost of the train. An analytical multidimensional traffic model for metro is proposed. The objective function is non-linear and written as the Lagrange function. The optimization problem is solved using Lagrangian duality theory which contains constraints including passenger load and dwell time equations.

By reducing departure time of the consecutive vehicle at the control station the APWT at the control station is minimized. The method is based on the minimization of the sum of headways, i.e. on the least squares principle [38]. The APWT, that randomly arrive at the station between $n$ vehicles in the observed period is equal to the sum of the square headways divided by the sum of headways during which the passenger arrives is

$$
A P W=\frac{\sum_{i=1}^{n}\left(D_{i}-D_{i-1}\right)^{2}}{2 \sum_{i=1}^{n}\left(D_{i}-D_{i-1}\right)^{2}}
$$

In order to improve passenger satisfaction and service quality in transportation system, the model for minimizing the total cost of the entire system is created. The model includes: waiting for the vehicle, or second vehicle on which to be transferred, and the cost of transfer. In practice, passengers usually tend to avoid a transfer, and if it is unavoidable then want minimal waiting for the next vehicle - passenger satisfaction. It is a nonlinear optimization problem and the problem of vehicle scheduling and timetabling. It is solved using nonlinear programming (such as sequential quadratic programming) and evolutionary algorithms (genetic algorithms) as shown in [18, 39].

The optimization models for creating the best timetables are sensitive to requirements (demandsensitive timetables - unlimited capacity, capacity condition, creating a timetable for the peak and off-peak times) as shown in [40]. The mathematical model calculates the departure time of the vehicle from the station. Optimization model for minimizing the total PWT at the station considers the following assumptions and conditions: the vehicle speed, waiting at the station and trip are the same (for simplifying the model), passengers uniformly arrive at the station, the number of vehicles is limited, minimal headway is defined, the departure time of the last vehicle is defined, and passengers must be in the vehicle (e.g. in 20 minutes). The model is defined as mixed-integer programming problem and solved using the exact method - CPLEX (B\&B algorithm) for MIP models.

The deterministic headways and entries in the buses with different capacities are observed in [41]. Multicriteria optimization methodology is proposed in two models. The uniform passenger arrival at the station is used in most papers. The first model minimizes headway deviations and reduces the expected waiting time of passengers that arrive to the station randomly. Heuristics algorithms are proposed for solving the latter problem.

\section{DISCUSSION}

After presenting the theoretical background, a discussion is performed. The correct algorithm selection for solving the problem of passenger transport depends on the execution time of the algorithm.

If it is important to maximize the service value (population function, employment opportunities, etc.) rather than minimizing the cost/distance of the trip, the problem of linear programming such as transit route arcnode service maximization (TRANSMax) problem is used [12]. The problem determines the optimum number of combinations of nodes and arcs that provide the best transport route.

Minimizing the PWT at the station in the entire network can be observed from two different points of view: even/uneven headway, regularity stop at each station or express train stop (stops only at some station in peak hour)

Mathematical methods that can manage the complex relationships between the PWT and time-dependent requests are proposed in [42]. Mixed-integer programming models compatible with high-ranking optimization platforms are defined. General Algebraic Modelling System (GAMS) and the appropriate package (AMPL) are used for modelling linear, nonlinear and mixed-integer optimisation problems.

From the hypothesis of a uniform arrival of passengers at the station the waiting time for the next vehicle is half of the headway. The exact mathematical formulation of the APWT at the station as opposed to other papers that consider uniform distribution of passenger arrival at the station regardless of the arrival of the vehicle is proposed in [43].

The decision making process whether to wait or not for the following vehicle that is late, in order to keep the time interval between vehicles, is named delay management.

Metaheuristic and exact algorithms are the most commonly used algorithms to solve the problem of vehicle delays in order to minimize the PWT at the station, to minimize the total travel time and for defining an optimum schedule that minimizes the total delay of vehicles [44-49].

If the optimization problem in the transport of passengers is considered as a problem of linear planning, the cost oriented or service-oriented problem is defined 
[50]. The problem of linear planning refers to the transport infrastructure, the travel time of passengers and selecting the best direction of travel and/or their frequency of use. If the problem is defined as a serviceoriented line planning and timetabling problem
(SOLPTP), it can be solved using a heuristic approach or cross-entropy metaheuristic method as presented in [50].

The problem of linear planning can also be formulated as a mixed integer linear program (MILP) and the exact methods such as $\mathrm{B} \& \mathrm{~B}$ and branch and cut are used [50].

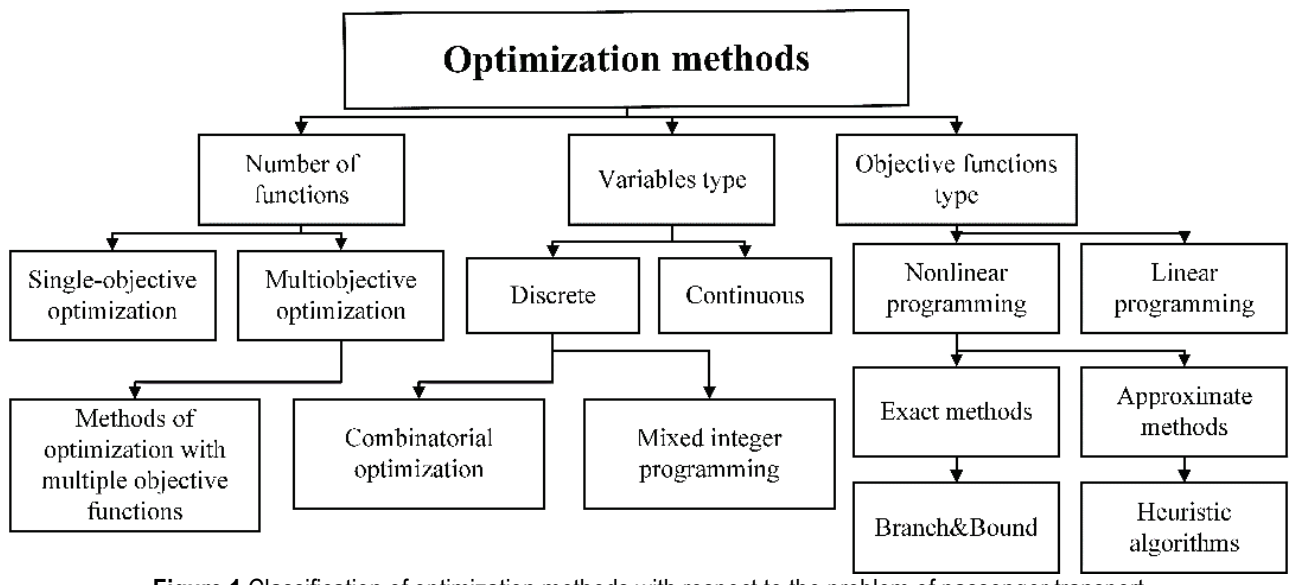

Figure 1 Classification of optimization methods with respect to the problem of passenger transport

Table 1 An overview of the related problems, objective functions and algorithms

\begin{tabular}{|c|c|c|c|}
\hline Autor(s) & Problem & Objective function & Algorithm/method/approach \\
\hline Aksu and Akyol [39] & Timetabling & Minimize the total system cost & $\begin{array}{l}\text { Genetic algorithm that creates clusters of routes } \\
\text { whose coordination reduces the transfer time } \\
\text { for connecting passengers }\end{array}$ \\
\hline Baita et al. [15] & $\begin{array}{l}\text { Vehicle scheduling problem } \\
\text { (VSP) }\end{array}$ & Minimizing the number of vehicles & Genetic algorithm \\
\hline Canca et la. [51] & Routing, Scheduling & $\begin{array}{l}\text { Minimization of the maximum consecutive } \\
\text { delay }\end{array}$ & Tabu search algorithm \\
\hline Corman et al. [44] & Train scheduling problem & Minimizing the total average waiting time & Mixed-integer nonlinear programming \\
\hline Dollevoet et al. [46] & Delay management problem & Minimize the total delay & Polynomial algorithm; CPLEX \\
\hline Ginkel and Schobel [47] & $\begin{array}{l}\text { Timetable; delay management } \\
\text { problem }\end{array}$ & Minimizing the sum of all delays & Exact algorithms \\
\hline $\begin{array}{l}\text { Ibarra-Rojas, Giesen } \\
\text { and Rios-Solis [52] }\end{array}$ & Timetable; scheduling & $\begin{array}{l}\text { Minimize the operating cost and maximize } \\
\text { number of passengers benefited }\end{array}$ & $\varepsilon$-constraint algorithm \\
\hline Kang et al. [53] & Last-train timetable & $\begin{array}{l}\text { Maximize the connection headway of } \\
\text { transferring passengers }\end{array}$ & Genetic algorithm \\
\hline Sun et al. [40] & Timetable & Minimize the total PWT & CPLEX (Branch-and-Bound) \\
\hline Wu et al. [26] & Timetable & Minimize the transfer waiting time & Genetic algorithm \\
\hline Yang, Li and Gao [54] & Timetable & $\begin{array}{l}\text { Minimize total passengers' time and the } \\
\text { total delay time }\end{array}$ & Branch and bound algorithm \\
\hline
\end{tabular}

Some of the classification criteria of optimization methods are: the number of objective functions, the type of variable and type of objective function. As presented in current literature, optimization problem of passenger transport can be classified as shown in Fig. 1.

The problem of transportation of passengers can be considered as single- or multicriteria discrete optimization, depending on the number of objective functions that are observed and the problem is solved using nonlinear programming with exact or approximate methods, depending on objective function (see Fig. 1).

Tab. 1 presents an overview of transportation problems, objective functions and algorithms dealing with the timetabling problem, what clarifies that the above mentioned research is focused mainly on the minimization of the total delay and transferring waiting time.

From the presented discussion, it can be concluded that the main objectives in solving the timetabling problem are the requirements and satisfaction of passengers, passenger waiting time and capacity of vehicles. The most commonly used algorithms for solving the problem that considers the latter objectives are $\mathrm{B} \& \mathrm{~B}$ for linear programming, and genetic algorithm for multiobjective optimization and nonlinear programming.

\section{CONCLUSION}

The problem of constructing the appropriate timetable in urban and intercity transport is the most widespread and most commonly solved problem in the field of passenger transport. Due to the globalization and increased use of public transportation arises the problem of capacity utilization of vehicles what additionally increases the costs of transport companies. The additional requirement in a modern transportation system is passenger satisfaction, i.e. the aim to carry passengers as soon as possible from one destination to another with a minimum waiting time at the station.

The paper presents an extensive overview of the models for solving the problem of transportation of passengers and for defining the optimal schedule for the public transport of passengers (urban or intercity) with different objective functions and constraints with possible 
algorithms for solving the presented problems. Despite the fact that the definition of the timetable is a very complex task, the paper clarifies that the requirements and satisfaction of passengers, passenger waiting time and capacity of vehicles are the main objectives in creating the timetable. The latter fact should focus future research on the already mentioned objectives as the most important to observe. The most commonly used algorithms are branch and bound and genetic algorithms.

Our future efforts are directed to the research of possible improvements in the presented models and algorithms. Additionally, we will consider the multicriteria optimization, i.e. observe satisfaction of passengers (to maximize satisfaction) and operating costs (to minimize expenses) at the same time.

\section{REFERENCES}

[1] Ceder, A. A. (1984). Bus frequency determination using passenger count data. Transportation Research Part A, 18(5-6). https://doi.org/10.1016/0191-2607(84)90019-0

[2] Ceder, A. \& Wilson, N. H. M. (1986). Bus network design. Transportation Research Part B, 20(4), 331-344. https://doi.org/10.1016/0191-2615(86)90047-0

[3] Ceder, A. A. (1987). Methods for creating bus timetables. Transportation Research Part A: Policy and Practice, 21(1), 59-83. https://doi.org/10.1016/0191-2607(87)90024-0

[4] Caprara, A., Fischetti, M., \& Toth, P. (2002). Modeling and Solving the Train Timetabling Problem. Operations Research, 50(5), 851-861. https://doi.org/10.1287/opre.50.5.851.362

[5] Siebert, M. \& Goerigk, M. (2013). An experimental comparison of periodic timetabling models. Computers \& Operations Research, 40(10), 2251-2259. https://doi.org/10.1016/j.cor.2013.04.002

[6] Shafia, M. A., Sadjadi, S. J., Jamili, A., TavakkoliMoghaddam, R., \& Pourseyed-Aghaee, M. (2012). The periodicity and robustness in a single-track train scheduling problem. Applied Soft Computing Journal, 12(1), 440-452. https://doi.org/10.1016/j.asoc.2011.08.026

[7] Bussieck, M. R., Kreuzer, P., \& Zimmermann, U. T. (1997). Optimal lines for railway systems. European Journal of Operational Research, 96(1), 54-63. https://doi.org/10.1016/0377-2217(95)00367-3

[8] Yalcinkaya, Ö. \& Bayhan, G. M. (2009). Modelling and optimization of average travel time for a metro line by simulation and response surface methodology. European Journal of Operational Research, 196, 225-233. https://doi.org/10.1016/j.ejor.2008.03.010

[9] Ceder, A. A., Golany, B., \& Tal, O. (2001). Creating bus timetables with maximal synchronization. Transportation Research Part A: Policy and Practice, 35, 913-928. https://doi.org/10.1016/S0965-8564(00)00032-X

[10] Ceder, A. A. (2007). Public Transit Planning and operation: Theory, modelling and practice. Oxford, UK: Butterworth-Heinemann.

[11] Jonaitis, J. (2007). Planning of the amount of trains needed for transportation by rail. Transport, 22(2), 83-89.

[12] Curtin, K. M. \& Biba, S. (2011). The Transit Route ArcNode Service Maximization problem. European Journal of Operational Research, 208(1), 46-56. https://doi.org/10.1016/j.ejor.2010.07.026

[13] Caprara, A., Kroon, L., \& Toth, P. (2010). Optimization Problems in Passenger Railway Systems. Wiley Encyclopedia of Operations Research and Management Science, J. J. Cochran, Ed. John Wiley \& Sons, 3896-3905.

[14] Chang, S. K. J. \& Hsu, S. C. (2003). Modeling of Passenger Waiting Time in Intermodal Station with Constrained
Capacity on Intercity Transit. Journal of the Eastern Asia Society for Transportation Studies, 5, 9-22.

[15] Baita, F., Pesenti, R., Ukovich, W., \& Favaretto, D. (2000). A comparison of different solution approaches to the vehicle scheduling problem in a practical case. Computers and Operations Research, 27(13), 1249-1269. https://doi.org/10.1016/S0305-0548(99)00073-8

[16] Herbon, A. \& Hadas, Y. (2015). Determining optimal frequency and vehicle capacity for public transit routes: A generalized newsvendor model. Transportation Research Part B: Methodological, 71, 85-99. https://doi.org/10.1016/j.trb.2014.10.007

[17] Hamdouch, Y., Ho, H. W., Sumalee, A., \& Wang, G. (2011). Schedule-based transit assignment model with vehicle capacity and seat availability. Transportation Research Part B: Methodological, 45(10), 1805-1830. https://doi.org/10.1016/j.trb.2011.07.010

[18] Wang, Y., Tang, T., Ning, B., van den Boom, T. J. J., \& De Schutter, B. (2015). Passenger-demands-oriented train scheduling for an urban rail transit network. Transportation Research Part C: Emerging Technologies, 60, 1-23. https://doi.org/10.1016/j.trc.2015.07.012

[19] Hassold, S. \& Ceder, A. A. (2012). Multiobjective Approach to Creating Bus Timetables with Multiple Vehicle Types. Transportation Research Record: Journal of the Transportation Research Board, 2276, 56-62. https://doi.org/10.3141/2276-07

[20] Hassold, S. \& Ceder, A. A. (2014). Public transport vehicle scheduling featuring multiple vehicle types. Transportation Research Part B: Methodological, 67, 129-143. https://doi.org/10.1016/j.trb.2014.04.009

[21] Ibarra-Rojas, O. J. \& Rios-Solis, Y. A. (2012). Synchronization of bus timetabling. Transportation Research Part B: Methodological, 46(5), 599-614. https://doi.org/10.1016/j.trb.2012.01.006

[22] Wong, R. C. W., Yuen, T. W. Y., Fung, K. W., \& Leung, J. M. Y. (2008). Optimizing Timetable Synchronization for Rail Mass Transit. Transportation Science, 42(1), 57-69. https://doi.org/10.1287/trsc. 1070.0200

[23] Shafahi, Y. \& Khani, A. (2010). A practical model for transfer optimization in a transit network: Model formulations and solutions. Transportation Research Part A: Policy and Practice, 44(6), 377-389. https://doi.org/10.1016/j.tra.2010.03.007

[24] Zhong, J. H., Shen, M., Zhang, J., Chung, H. S. H., Shi, Y. H., \& Li, Y. (2013). A differential evolution algorithm with dual populations for solving periodic railway timetable scheduling problem. IEEE Transactions on Evolutionary Computation, 17, 512-527. https://doi.org/10.1109/TEVC.2012.2206394

[25] Wu, Y., Tang, J., Yu, Y., \& Pan, Z. (2015). A stochastic optimization model for transit network timetable design to mitigate the randomness of traveling time by adding slack time. Transportation Research Part C: Emerging Technologies, 52, 15-31. https://doi.org/10.1016/j.trc.2014.12.012

[26] Wu, J., Liu, M., Sun, H., Li, T., Gao, Z., \& Wang, D. Z. W. (2015). Equity-based timetable synchronization optimization in urban subway network. Transportation Research Part C: Emerging Technologies, 51, 1-18. https://doi.org/10.1016/j.trc.2014.11.001

[27] Niu, H. \& Zhang, M. (2012). An Optimization to Schedule Train Operations with Phase-Regular Framework for Intercity Rail Lines. Discrete Dynamics in Nature and Society, 2012, 1-13. https://doi.org/10.1155/2012/549374

[28] Barrena, E., Canca, D., Coelho, L. C., \& Laporte, G. (2014). Exact formulations and algorithm for the train timetabling problem with dynamic demand. Computers and Operations Research, 44, 66-74. https://doi.org/10.1016/j.cor.2013.11.003 
[29] Barrena, E., Canca, D., Coelho, L. C., \& Laporte, G. (2014). Single-line rail rapid transit timetabling under dynamic passenger demand. Transportation Research Part B: Methodological, 70, 134-150. https://doi.org/10.1016/j.trb.2014.08.013

[30] Nachtigall, K. \& Voget, S. (1997). Minimizing waiting times in integrated fixed interval timetables by upgrading railway tracks. European Journal of Operational Research, 2217(96), 610-627. https://doi.org/10.1016/S0377-2217(96)00284-6

[31] Nachtigall, K. \& Voget, S. (1996). A genetic algorithm approach to periodic railway synchronization. Computers and Operations Research, 23(5), 453-463. https://doi.org/10.1016/0305-0548(95)00032-1

[32] Yang, X., Ning, B., Li, X., \& Tang, T. (2014). A TwoObjective Timetable Optimization Model in Subway Systems. IEEE Transactions on Intelligent Transportation Systems, 15(5), 1913-1921. https://doi.org/10.1109/TITS.2014.2303146

[33] Vansteenwegen, P. \& Van Oudheusden, D. (2006). Developing railway timetables which guarantee a better service. European Journal of Operational Research, 173, 337-350. https://doi.org/10.1016/j.ejor.2004.12.013

[34] Vansteenwegen, P. \& Van Oudheusden, D. (2007). Decreasing the passenger waiting time for an intercity rail network. Transportation Research Part B: Methodological, 41(4), 478-492. https://doi.org/10.1016/j.trb.2006.06.006

[35] Corman, F., D’Ariano, A., Pacciarelli, D., \& Pranzo, M. (2012). Bi-objective conflict detection and resolution in railway traffic management. Transportation Research Part C: Emerging Technologies, 20(1), 79-94. https://doi.org/10.1016/.t.trc.2010.09.009

[36] Parbo, J., Nielsen, O. A., \& Prato, C. G. (2014). User perspectives in public transport timetable optimisation. Transportation Research Part C: Emerging Technologies, 48(2014), 269-284. https://doi.org/10.1016/j.trc.2014.09.005

[37] Sun, X., Zhang, S., \& Dong, H. (2015). Optimization of Metro Train Schedules with a Dwell Time Model Using the Lagrangian Duality Theory. IEEE Transactions on Intelligent Transportation Systems, 16(3), 1285-1293. https://doi.org/10.1109/TITS.2014.2361894

[38] Berrebi, S. J., Watkins, K. E., \& Laval, J. A. (2015). A realtime bus dispatching policy to minimize passenger wait on a high frequency route. Transportation Research Part B: Methodological, 81(2), 377-389. https://doi.org/10.1016/j.trb.2015.05.012

[39] Aksu, D. T. \& Akyol, U. (2014). Transit Coordination Using Integer-Ratio Headways. IEEE Transactions on Intelligent Transportation Systems, 15(4), 1633-1642. https://doi.org/10.1109/TITS.2014.2301821

[40] Sun, L., Jin, J. G., Lee, D. H., Axhausen, K. W., \& Erath, A. (2014). Demand-driven timetable design for metro services. Transportation Research Part C: Emerging Technologies, 46, 284-299. https://doi.org/10.1016/..trc.2014.06.003

[41] Ceder, A. A., Hassold, S., \& Dano, B. (2013). Approaching even-load and even-headway transit timetables using different bus sizes. Public Transport, 5(3), 193-217. https://doi.org/10.1007/s12469-013-0062-z

[42] Niu, H., Zhou, X., \& Gao, R. (2015). Train scheduling for minimizing passenger waiting time with time-dependent demand and skip-stop patterns: Nonlinear integer programming models with linear constraints. Transportation Research Part B: Methodological, 76, 117135. https://doi.org/10.1016/j.trb.2015.03.004

[43] Amin-Naseri, M. R. \& Baradaran, V. (2014). Accurate Estimation of Average Waiting Time in Public Transportation Systems. Transportation Science, 49(2), 213-222. https://doi.org/10.1287/trsc.2013.0514

[44] Corman, F., D’Ariano, A., Pacciarelli, D., \& Pranzo, M.
(2010). A tabu search algorithm for rerouting trains during rail operations. Transportation Research Part B: Methodological, 44(1), 175-192. https://doi.org/10.1016/j.trb.2009.05.004

[45] Heilporn, G., De Giovanni, L., \& Labbé, M. (2008). Optimization models for the single delay management problem in public transportation. European Journal of Operational Research, 189(3), 762-774. https://doi.org/10.1016/j.ejor.2006.10.065

[46] Dollevoet, T., Huisman, D., Schmidt, M., \& Schobel, A. (2012). Delay Management with Rerouting of Passengers. Transportation Science, 46(1), 74-89. https://doi.org/10.1287/trsc.1110.0375

[47] Ginkel, A. \& Schobel, A. (2007). To Wait or Not to Wait? The Bicriteria Delay Management Problem in Public Transportation. Transportation Science, 41, 527-538. https://doi.org/10.1287/trsc.1070.0212

[48] Mu, S. \& Dessouky, M. (2011). Scheduling freight trains traveling on complex networks. Transportation Research Part B: Methodological, 45(7), 1103-1123. https://doi.org/10.1016/j.trb.2011.05.021

[49] Schachtebeck, M. \& Schobel, A. (2010). To Wait or Not to Wait--And Who Goes First? Delay Management with Priority Decisions. Transportation Science, 44(3), 307-321. https://doi.org/10.1287/trsc. 1100.0318

[50] Kaspi, M. \& Raviv, T. (2013). Service-Oriented Line Planning and Timetabling for Passenger Trains. Transportation Science, 47(3), 295-311. https://doi.org/10.1287/trsc.1120.0424

[51] Canca, D., Zarzo, A., Algaba, E., \& Barrena, E. (2011). Confrontation of different objectives in the determination of train scheduling. Procedia - Social and Behavioral Sciences, 20, 302-312. ttps://doi.org/10.1016/j.sbspro.2011.08.036

[52] Ibarra-Rojas, O. J., Giesen, R., \& Rios-Solis, Y. A. (2014). An integrated approach for timetabling and vehicle scheduling problems to analyze the trade-off between level of service and operating costs of transit networks. Transportation Research Part B: Methodological, 70, 3546. https://doi.org/10.1016/j.trb.2014.08.010

[53] Kang, L., Wu, J., Sun, H., Zhu, X., \& Gao, Z. (2015). A case study on the coordination of last trains for the Beijing subway network. Transportation Research Part B: Methodological, 72, 112-127. ttps://doi.org/10.1016/j.trb.2014.09.003

[54] Yang, L., Li, K., \& Gao, Z. (2009). Train timetable problem on a single-line railway with fuzzy passenger demand. IEEE Transactions on Fuzzy Systems, 17(3), 617-629. https://doi.org/10.1109/TFUZZ.2008.924198

\section{Contact information}

Ivana HARTMANN Tolić, MSc

Goran MARTINOVĆ, PhD, Full Professor in Computer Science

Dominika CRNJAC-MILIĆ, PhD, Associate Professor

Faculty of Electrical Engineering, Computer Science and Information

Technology Osijek, Josip Juraj Strossmayer University of Osijek, Croatia

Kneza Trpimira 2b, 31000 Osijek, Croatia

E-mail: ivana.hartmann@ferit.hr

E-mail: goran.martinovic@ferit.hr

E-mail: dominika.crnjac@ferit.hr 